\title{
Appearances are Deceptive: Staphylococcus Superinfection of Clavicular Tuberculous Osteomyelitis
}

\author{
Narendranath Epperla, MD; Siddhartha Kattamanchi, MD; and Thomas R. Fritsche, MD, PhD
}

\begin{abstract}
A man, aged 25 years, presented with pain, swelling, and drainage from the right clavicular area. $\mathrm{He}$ had a past history of abscess at the sternoclavicular joint. The cultures from the drainage site grew methicillin-sensitive Staphylococcus aureus, and he was placed on appropriate antibiotics. As S. aureus infection of the clavicle is often secondary in nature, particularly in adults, a thorough workup was done to identify the underlying cause. Quantiferon gold, done as a part of the workup, came back positive, while the bone cultures grew S. aureus and Mycobacterium tuberculosis. He was placed on 9 months of combination therapy for tuberculosis osteomyelitis with a good clinical outcome.
\end{abstract}

Keywords: Clavicle; Tuberculous osteomyelitis; Staphylococcus aureus

\author{
Corresponding Author: \\ Narendranath Epperla, MD \\ Medical College of Wisconsin \\ Department of Hematology-Oncology \\ 9200 W. Wisconsin Ave. \\ Milwaukee, WI 53226 \\ Tel: (4I4) 805-4600 \\ Email: nepperla@mcw.edu \\ Received: April 29, 2014 \\ Revised: July I, 2014 \\ Accepted: July 24, 20I4 \\ doi: $10.3121 / \mathrm{cmr} .2014 .1247$
}

$\mathrm{T}$

uberculous osteomyelitis with staphylococcal super-infection is a rare presentation of clavicular osteomyelitis. This case highlights the importance of ruling out secondary causes like tuberculosis (TB) when staphylococcal infection of the clavicle is encountered.

\section{Case Presentation}

A man, aged 25 years, presented with pain, swelling, and drainage from the right clavicular area (figure 1) of 15 days duration, limiting his activity. Four months earlier, he had been diagnosed with an abscess at the right sternoclavicular joint at an outside facility for which he underwent incision and drainage (I\&D). The drainage fluid was not sent for routine (aerobic and anerobic) cultures or mycobacterium species. After the procedure, he was doing relatively well until the current presentation. It was noted that he had two episodes of cough with hemoptysis along with a 5-pound weight loss in the last 6 months. He denied any history of trauma to the clavicular or overlying skin area. He reported no history of irradiation, diabetes mellitus, intravenous drug use or known or suspected HIV exposure. He had resided in India until his late teens, when he moved to Poland to continue his education (3 years), after which he moved to the United States. His father had tuberculosis 20 years prior and had been treated with antitubercular therapy without relapse.

Physical examination was normal except for the presence of mild erythema and tenderness at the right clavicular region at the drainage site (figure 1). Two sets of blood cultures were drawn, and he was placed on empiric antibiotic coverage, starting with intravenous vancomycin (1 gram every 8 hours). This therapy was 


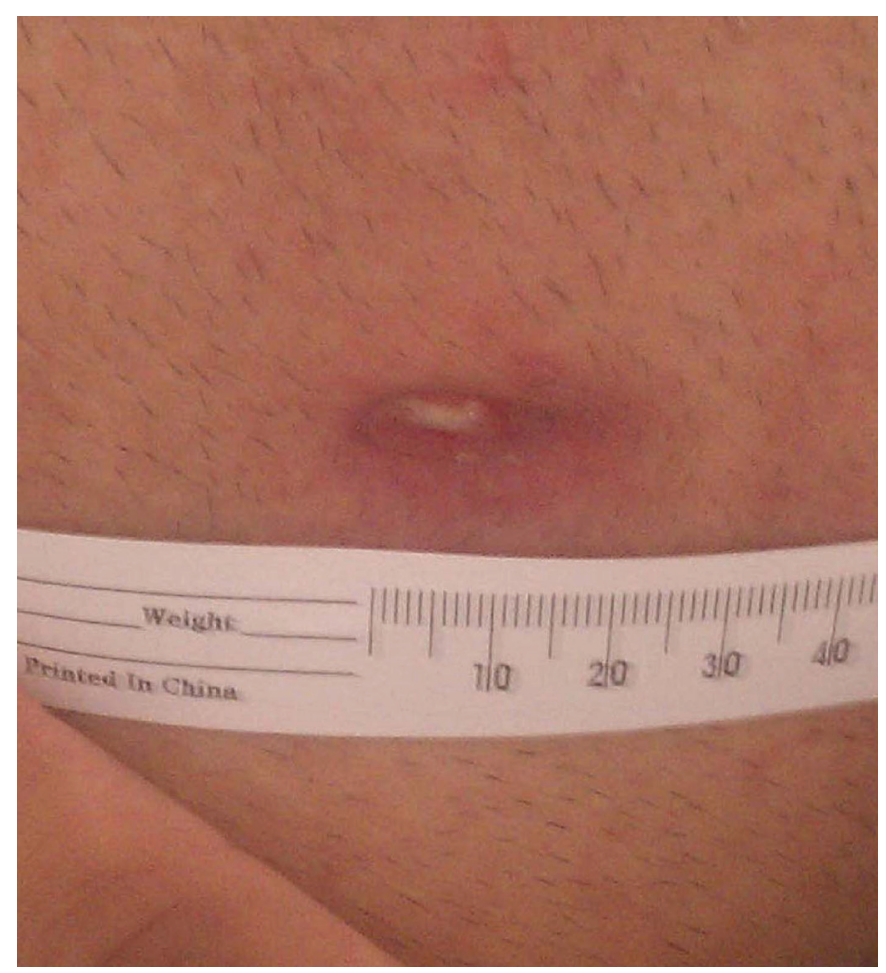

Figure 1. Discharging sinus over the right clavicle measuring approximately $1.5 \mathrm{~cm}$.

changed to oral cephalexin ( $750 \mathrm{mg}$ three times daily) when culture from the drainage site was positive for methicillinsensitive Staphylococcus aureus (S. aureus). Of note, both sets of blood cultures came back negative. As clavicular infection with $S$. aureus is rare and usually secondary to a predisposing factor, efforts were made to identify the underlying etiology.

A chest radiograph was performed and found to be normal. Further imaging studies in the form of computed tomography (CT) scans of the chest were pursued and revealed two lytic lesions in the medial right clavicle along with mediastinal and hilar lymphadenopathy (figure $2 \mathrm{a} \& 2 \mathrm{~b}$ ). The patient underwent excision of the sinus tract along with a clavicular bone biopsy. The latter was sent for microbiology studies that grew $S$. aureus in the routine aerobic culture and Mycobacterium tuberculosis (TB) in the Mycobacteria Growth Indicator Tube (MGIT). Simultaneously the Quantiferon Gold assay for tuberculosis was positive and the HIV test was negative. In our patient, it is likely that the $S$. aureus infection was due to the open, draining fistula that developed as a result of the initial procedure (I\&D), permitting ingress of $S$. aureus, leading to the staphylococcal superinfection.

The patient was started on 9 months of combination therapy (isoniazid, rifampin, pyrazinamide, and ethambutol for 2 months, then isoniazid and rifampin for 7 months) for TB osteomyelitis. He was then followed through teleconference, every 3 months, until completion of treatment. He continues to do well with no recurrence of symptoms.

\section{Discussion}

Despite the advent of anti-tubercular drugs in 1940, the number of TB cases remains high worldwide (with an estimated 8.3 million incident cases and 1.3 million deaths from TB in 2012 $)$; however, there has been a slow but gradual decline in TB cases since 2001 (the rate of decline was $2 \%$ between 2011 and 2012). ${ }^{1}$ Of note, the incidence of $\mathrm{TB}$ in the United States remains very low (estimated new cases of TB [all forms] per 100,000 population is $0-9.9),{ }^{1}$ which has led physicians to lower their index of suspicion for this disease. Though uncommon, physicians from regions of low TB endemicity could encounter unusual presentations of $\mathrm{TB}$, and hence, should have increased clinical awareness of both pulmonary and extra-pulmonary manifestations of TB.

Osteoarticular/skeletal TB constitutes $1 \%-4.5 \%$ of all TB cases and $5 \%-15 \%$ of all extrapulmonary TB cases..$^{2-5}$ Watts and Lifeso ${ }^{6}$ describe the spine as the most common site for osteoarticular TB $(50 \%$ thoracic spine, $25 \%$ cervical spine, $25 \%$ lumbar spine) followed by the pelvis (12\%), hip and femoral bone $(10 \%)$, ribs $(7 \%)$, ankle, shoulder, elbow and wrist $(2 \%){ }^{6}$ Clavicular TB is seen in $<1 \%$ of cases with osteoarticular TB. ${ }^{7}$ A superimposed staphylococcal infection on a clavicle infected with TB is a rare presentation, with only a few cases reported to date.

Osteomyelitis of the clavicle is a rare condition accounting for $0 \%$ to $7 \%$ of all acute osteomyelitis cases, with incidence ranging from $0 \%$ in a mixed-age population to $7 \%$ in children. ${ }^{8-10}$ The medial half of the clavicle seems to be the most commonly affected site, although any portion may be affected. ${ }^{10-12}$ Osteomyelitis of the clavicle can occur due to hematogenous or contiguous spread as well as direct inoculation. ${ }^{12}$ In children, its origin is generally hematogenous, while in adults, clavicular osteomyelitis is more commonly seen in association with a predisposing factor, such as systemic coccidioidomycosis, intravenous drug abuse, TB, mitral valve prosthesis, diabetes, previous lung carcinoma, or prior head and neck surgical procedures. ${ }^{9-12} S$. aureus is the predominant causal agent in clavicular infections, and occurs in all age groups. ${ }^{10-12}$

Osteoarticular tuberculosis is thought to be due to the reactivation of dormant organisms. ${ }^{11-13}$ The risk factors for the development of TB include recent TB contact, emigration from endemic areas, previous pulmonary TB, HIV infection, lower socioeconomic class, homelessness, and imprisonment. ${ }^{11-13}$ Our patient had emigrated from India, making it possible that he had been infected in his country of origin and that this infection was subclinical, since he had no previous history of TB, and his chest radiograph was normal. Reactivation of TB may have occurred when he immigrated to the United States. Ustianowski et $\mathrm{al}^{14}$ suggested that the infection is reactivated when a person migrates to another country, and it manifests in a different organ for reasons that are unclear. 


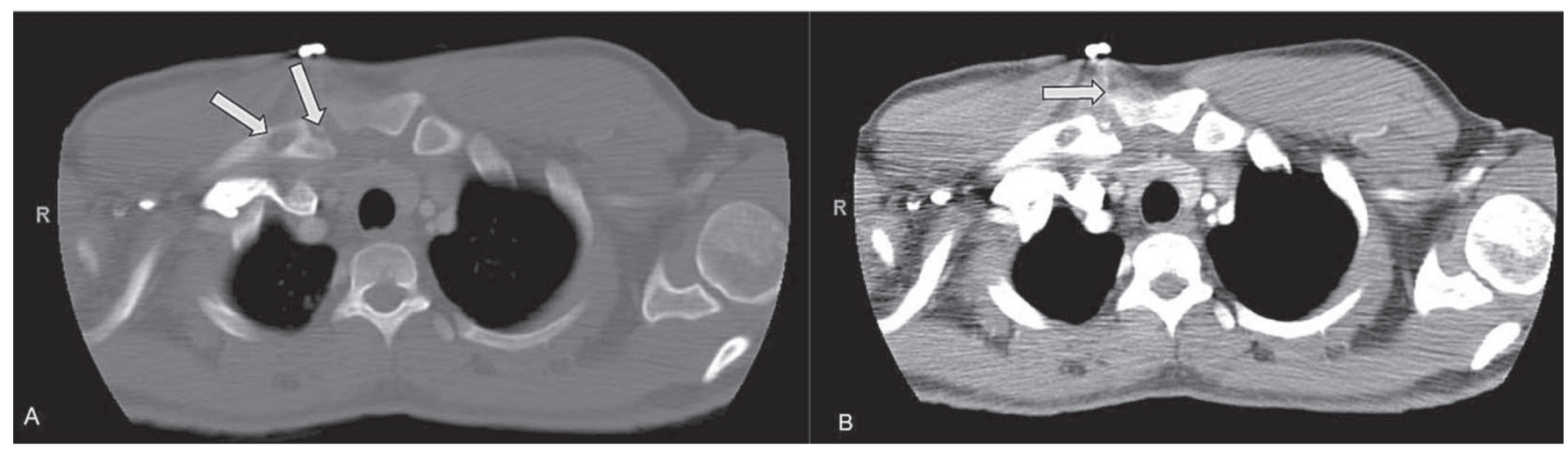

Figure 2. (A) Computed tomography (CT) scan of the chest in bone window setting, demonstrating the presence of lytic lesions on the medial aspect of the clavicle, as indicated by the arrows. (B) CT scan of the chest in abdominal window setting, showing the sinus tract and the presence of hilar lymphadenopathy, as indicated by the arrow.

The majority of patients with osteoarticular TB have solitary skeletal lesions. Multifocal lesions are seen in only $30 \%$ of cases. ${ }^{15,16}$ Rarely is there concomitant pulmonary TB. ${ }^{16}$ In the early stages, patients usually present with painful swelling of the clavicle, with no major bone destruction; only in the later stages is there formation of a cold abscess or sinuses/ulcers. Superinfection with $S$. aureus can complicate some cases of osteoarticular tuberculosis. ${ }^{17}$ These cases are often mistaken as isolated $S$. aureus osteomyelitis cases and treated as such, due to the fact that most of these patients will have a negative TB skin test and normal chest radiograph. There is slight improvement in symptoms after antibiotics, but they recur, and the infection continues its course, as in our patient. Therefore, a delay in diagnosis can lead to disease progression and the risk of devastating destruction of the affected bone or joint. Hence, it is very important to rule out predisposing factors like TB in patients who present with staphylococcal osteomyelitis of the clavicle. ${ }^{17,18}$

Osteoarticular TB is a pauci-bacillary disease; therefore, a TB skin test can be negative in many cases, and most patients will also have a normal radiograph. ${ }^{13}$ Histology and culture of the biopsy sample remain the gold standards for diagnosis, providing positive results in $>90 \%$ of cases. ${ }^{19}$ The tissue needed can be obtained from the sinus/ulcer edge, bone, or synovial biopsy. Polymerase chain reaction (PCR) has sensitivity and specificity of $62.5 \%$ and $91.66 \%$, respectively, for non-spinal samples; therefore a positive PCR would call for treatment, while a negative PCR requires further evaluation by histopathological examination. ${ }^{18,20}$ PCR also offers an advantage in that it can establish diagnosis within 24 hours, while culture results can take 6 to 8 weeks. Due to overlapping anatomic structures, radiographs are not conclusive in evaluating changes in the clavicle. ${ }^{11,21,22}$ Magnetic resonance imaging (MRI) or CT scans, on the other hand, provide far superior images. ${ }^{11,21,22}$ Lytic changes are better analyzed in CT scans, especially in the bone window setting, while MRI scans are effective tools in determining the extent of lesions, particularly when there is marrow and soft tissue involvement. All imaging methods, however, provide information complementing the clinical presentation. ${ }^{22}$
Standard management of osteoarticular TB includes treatment with 9 months of combination therapy (isoniazid, rifampin, pyrazinamide, and ethambutol for 2 months, followed by isoniazid and rifampin for 7 months). When diagnosed early, patients can be managed with anti-tubercular drugs alone. ${ }^{17}$ In cases of uncertain diagnosis, unresponsive disease, or for the removal of a large sequestrum, surgical excision may be necessary. ${ }^{23}$ The regeneration of the resected segment of the clavicle is often seen in young children, but rarely occurs in adults. Most patients who have had total or partial clavicular resection have little or no residual instability or loss of motion in the affected shoulder following surgery. ${ }^{24}$

\section{Conclusion}

TB has a chronic and insidious course that could be masked by other bacterial co-infections. Clinicians should have a heightened sense of awareness for TB in cases of staphylococcal osteomyelitis of the clavicle. One should not hesitate to evaluate with imaging modalities, such as MRI and CT scans, and proceed early to an open biopsy, as delay in diagnosis can lead to disease progression and the risk of devastating destruction of the affected bone or joint.

\section{Acknowledgements}

The authors thank the Marshfield Clinic Research Foundation's Office of Scientific Writing and Publication for assistance with this case report.

\section{References}

1. World Health Organization. Global Tuberculosis Report 2013. Geneva, Switzerland: WHO Publications; 2013. Available at: http://apps.who.int/iris/bitstream/10665/91355/1/9789241564 656_eng.pdf?ua=1. Accessed June 19, 2014.

2. Davidson PT, Horowitz I. Skeletal tuberculosis: A review with patient presentations and discussion. Am J Med 1970; 48:77-84.

3. Jutte PC, van Loenhout-Rooyackers JH, Borgdorff MW, van Horn JR. Increase of bone and joint tuberculosis in the Netherlands. J Bone Joint Surg Br 2004;86:901-904.

4. Sharma SK, Mohan A. Extrapulmonary tuberculosis. Indian J Med Res 2004;120:316-353. 
5. Enache SD, Pleasea IE, Anusca D, Zaharia B, Pop OT. Osteoarticular tuberculosis-a ten years case review. Rom J Morphol Embryol 2005;46:67-72.

6. Watts HG, Lifeso RM. Tuberculosis of bones and joints. J Bone Joint Surg Am 1996;78:288-298.

7. Srivastava KK, Garg LD, Kochar VL. Tuberculous osteomyelitis of the clavicle. Acta Orthop Scand 1974; 45:668-672.

8. Gerszten E, Allison MJ, Dalton HP. An epidemiologic study of 100 consecutive cases of osteomyelitis. South Med J 1970;63:365-367.

9. Green M, Nyhan WL, Fousek MD. Acute hematogenous osteomyelitis. Pediatrics 1956;17:368-382.

10. Park HM, Rothschild PA, Kernek CB. Scintigraphic evaluation of extremity pain in children: its efficacy and pitfalls. AJR Am J Roentgenol 1985;145:1079-1084.

11. Gerscovich EO, Greenspan A. Osteomyelitis of the clavicle: clinical, radiologic, and bacteriologic findings in ten patients. Skeletal Radiol 1994;23:205-210.

12. Alessi DM, Sercarz JA, Calcaterra TC. Osteomyelitis of the clavicle. Arch Otolaryngol Head Neck Surg 1988; 114:1000-1002.

13. Sequeira W, Co H, Block JA. Osteoarticular tuberculosis: current diagnosis and treatment. Am J Ther 2000;7:393-398.

14. Ustianowski A, Shaffer R, Collin S, Wilkinson RJ, Davidson $\mathrm{RN}$. Prevalence and associations of vitamin D deficiency in foreign-born persons with tuberculosis in London. J Infect 2005;50:432-437.

15. Nathanson L, Cohen W. A statistical and roentgen analysis of two hundred cases of bone and joint tuberculosis. Radiology 1941;36:550-567.

16. Berney S, Goldstein M, Bishko F. Clinical and diagnostic features of tuberculous arthritis. Am J Med 1972;53:36-42.

17. Sinnott JT $4^{\text {th }}$, Cancio MR, Frankle MA, Gustke K, Spiegel PG. Tuberculous osteomyelitis masked by concomitant staphylococcal infection. Arch Intern Med 1990; 150:1865-1867.

18. Wallace R, Cohen AS. Tuberculosis arthritis: a report of two cases with review of biopsy and synovial fluid findings. Am J Med 1976;61:277-282.

19. Pandey V, Chawla K, Acharya K. Rao, S, Rao S. The role of polymerase chain reaction in the management of osteoarticular tuberculosis. Int Orthop 2009;33:801-805.

20. Negi SS, Gupta S, Khare S, Lal S. Comparison of various microbiological tests including polymerase chain reaction for the diagnosis of osteoarticular tuberculosis. Indian J Med Microbiol 2005;23:245-248.

21. Yasuda T, Tamura K, Fujiwara M. Tuberculous arthritis of the sternoclavicular joint . A report of three cases. J Bone Joint Surg Am 1995;77:136-139.

22. Shah J, Patkar D, Parikh B, Parmar H, Varma R, Patankar T, Prasad S. Tuberculosis of the sternum and clavicle: imaging findings in 15 patients. Skeletal Radiol 2000;29:447-453.

23. Tuli SM. Tuberculosis of the Skeletal System. $3^{\text {rd }}$ ed. New Delhi: Jaypee Brothers; 2004: pp. 167-173.

24. Kochhar VL, Srivastava KK. Anatomical and functional consideration in total claviclectomy. Clin Orthop Relat Res 1976;118:199-201.

\section{Author Affiliations}

Narendranath Epperla, MD ${ }^{*} ;$; Siddhartha Kattamanchi, $M D^{*}$; and Thomas R. Fritsche, $M D, P h D^{*}$

*Department of Internal Medicine, Marshfield Clinic, Marshfield WI, USA

Current affiliation: Department of Hematology-Oncology, Medical College of Wisconsin, Milwaukee, WI, USA

* Department of Laboratory Medicine, Marshfield Clinic, Marshfield WI, USA 